\title{
THE CLASS OF COMPLEX SYMMETRIC OPERATORS IS NOT NORM CLOSED
}

\author{
SEN ZHU, CHUN GUANG LI, AND YOU QING JI
}

(Communicated by Marius Junge)

\begin{abstract}
An operator $T \in \mathcal{B}(\mathcal{H})$ is complex symmetric if there exists a conjugate-linear, isometric involution $C: \mathcal{H} \longrightarrow \mathcal{H}$ so that $C T C=T^{*}$. In this paper, a class of complex symmetric operators on finite dimensional Hilbert spaces is constructed. As an application, it is shown that Kakutani's unilateral weighted shift operator is not complex symmetric; however, it is a norm limit of complex symmetric operators. This gives a negative answer to a question of S. Garcia and W. Wogen: that is, whether or not the class of complex symmetric operators is norm closed.
\end{abstract}

Throughout this paper, $\mathbb{C}$ and $\mathbb{N}$ denote the set of complex numbers and the set of positive integers respectively. $\mathcal{H}$ will always denote a complex separable infinite dimensional Hilbert space. We let $\mathcal{B}(\mathcal{H})$ denote the algebra of all bounded linear operators on $\mathcal{H}$.

Generalizing the notion of complex symmetric matrices, S. Garcia and M. Putinar 4 initiated a study for complex symmetric operators on Hilbert spaces which have many motivations in function theory, matrix analysis and other areas.

Definition 0.1. A conjugation is a conjugate-linear map $C: \mathcal{H} \longrightarrow \mathcal{H}$ which is both involutive (i.e., $\left.C^{2}=I\right)$ and isometric (i.e., $\left.(C x, C y)=(y, x), \forall x, y \in \mathcal{H}\right)$.

Definition 0.2. We say that an operator $T \in \mathcal{B}(\mathcal{H})$ is complex symmetric if there exists a conjugation $C$ on $\mathcal{H}$ so that $C T C=T^{*}$. Denote by $\mathcal{S}(\mathcal{H})$ the subset of $\mathcal{B}(\mathcal{H})$ consisting of all bounded complex symmetric operators on $\mathcal{H}$.

Through a series of papers [4, 5, 9, 6, 3, 7, 2, 8, 1, mathematicians have obtained a better understanding of the structure of complex symmetric operators. In fact, $\mathcal{S}(\mathcal{H})$ encompasses many of the well-known and useful classes of operators such as Hankel operators, truncated Toeplitz operators, normal operators and binormal operators.

In a recent paper [7, S. Garcia and W. Wogen proved that $\mathcal{S}(\mathcal{H})$ is not closed in the strong operator topology. Moreover, they raised the following norm closure problem.

Question 0.3. Is $\mathcal{S}(\mathcal{H})$ norm closed?

Received by the editors January 20, 2011.

2010 Mathematics Subject Classification. Primary 47A05; Secondary 47B99.

Key words and phrases. Complex symmetric operators, Kakutani's shift.

This work was supported by NNSF of China (11026038, 10971079, 11101177) and the Basic Research Foundation of Jilin University (201001001, 201103194). 
The aim of this short paper is to give a negative answer to the above question. In fact, by constructing a class of complex symmetric operators on finite dimensional Hilbert spaces, we shall prove that Kakutani's unilateral weighted shift is a norm limit of complex symmetric operators, but it is not complex symmetric.

If $\mathcal{K}$ is a complex Hilbert space and $e, f \in \mathcal{K}$, then we let $e \otimes f$ denote the following bounded linear operator on $\mathcal{K}$ :

$$
(e \otimes f)(x)=(x, f) e, \forall x \in \mathcal{K} .
$$

We first give a key result.

Proposition 0.4. Let $n \in \mathbb{N}$ and $\left\{e_{i}\right\}_{i=1}^{n}$ be an othonormal basis (ONB for short) of $\mathbb{C}^{n}$. Assume that

$$
T=\sum_{i=1}^{n-1} \lambda_{i} e_{i} \otimes e_{i+1},
$$

where $\lambda_{i}=\lambda_{n-i}$ for all $1 \leq i \leq n-1$. Then $T$ is a complex symmetric operator.

Proof. For $x \in \mathbb{C}^{n}, x=\sum_{i=1}^{n} \alpha_{i} e_{i}$, define $C x=\sum_{i=1}^{n} \overline{\alpha_{i}} e_{n-i+1}$. Obviously, $C$ is a conjugation on $\mathbb{C}^{n}$ and $C e_{i}=e_{n-i+1}$ for $1 \leq i \leq n$. It suffices to verify that $C T C=T^{*}$. Since $C T C$ and $T^{*}$ are both linear on $\mathbb{C}^{n}$, we need only prove that $C T C e_{i}=T^{*} e_{i}$ for all $i$.

First, one can note that

$$
T^{*}=\sum_{i=1}^{n-1} \overline{\lambda_{i}} e_{i+1} \otimes e_{i} .
$$

Then $C T C e_{n}=C T e_{1}=0=T^{*} e_{n}$.

Let $i(1 \leq i \leq n-1)$ be fixed. Then, by definition of $T$,

$$
C T C e_{i}=C T e_{n-i+1}=C\left(\lambda_{n-i} e_{n-i}\right)=\overline{\lambda_{n-i}} e_{i+1},
$$

and, by definition of $T^{*}$,

$$
T^{*} e_{i}=\overline{\lambda_{i}} e_{i+1} .
$$

Note that $\lambda_{i}=\lambda_{n-i}$; thus we have $C T C e_{i}=T^{*} e_{i}$. This completes the proof.

Let $W$ denote Kakutani's unilateral weighted shift ([10, page 282]), defined by $W e_{n}=a_{n} e_{n+1}$ with respect to the orthonormal basis $\left\{e_{n}\right\}_{n=1}^{\infty}$ of $\mathcal{H}$, where $a_{n}=\frac{1}{2^{k-1}}$ and $k$ is the exponent occurring in the (unique) decomposition $n=$ $2^{k-1}(2 m-1), k, m \in \mathbb{N}$.

Theorem 0.5. Let $W \in \mathcal{B}(\mathcal{H})$ be defined as above. Then, given $\varepsilon>0$, there exists a complex symmetric operator $T \in \mathcal{B}(\mathcal{H})$ such that $\|W-T\|<\varepsilon$.

Proof. For given $\varepsilon>0$, there exists $k \in \mathbb{N}$ such that $\frac{1}{2^{k}}<\varepsilon$. We may assume that $k>1$. Denote $E=\left\{2^{l-1}(2 m-1): l, m \in \mathbb{N}\right.$ and $\left.1 \leq l \leq k\right\}$. Then $\mathbb{N} \backslash E$ is in fact the set of all positive integers $n$ which admit $2^{k}$ as a divisor.

For each $i \in \mathbb{N}$, set

$$
b_{i}= \begin{cases}a_{i}, & i \in E, \\ 0, & i \in \mathbb{N} \backslash E\end{cases}
$$

and

$$
T=\sum_{i=1}^{\infty} b_{i} e_{i+1} \otimes e_{i} .
$$


Then it is easy to see that $T$ is a unilateral weighted shift with weights $\left\{b_{i}\right\}_{i=1}^{\infty}$ and $\|W-T\|<\varepsilon$. It suffices to prove that $T$ is a complex symmetric operator.

We claim that (i) $b_{m}=b_{2^{k}+m}$ for all $m \in \mathbb{N}$ and (ii) $b_{i}=b_{2^{k}-i}$ for all $1 \leq i \leq$ $2^{k}-1$.

Let $m \in \mathbb{N}$ be fixed. If $2^{k}$ divides $m$, then it also divides $2^{k}+m$. Then, by definition, $b_{m}=0=b_{2^{k}+m}$. If $2^{k}$ does not divide $m$, then there exists $1 \leq l \leq k$ and an odd $p \in \mathbb{N}$ such that $m=2^{l-1} p$. Thus $2^{k}+m=2^{k}+2^{l-1} p=2^{l-1}\left(2^{k-l+1}+p\right)$. Note that $2^{k-l+1}+p$ is odd; then $2^{k}$ does not divide $2^{k}+m$ and

$$
b_{m}=a_{m}=\frac{1}{2^{l-1}}=a_{2^{l-1}\left(2^{k-l+1}+p\right)}=a_{2^{k}+m}=b_{2^{k}+m} .
$$

Let $1 \leq i \leq 2^{k}-1$ be fixed. Obviously $i$ can be written as $i=2^{j-1} p$, where $j, p \in \mathbb{N}, 1 \leq j \leq k$, and $p$ is odd. Then $2^{k}-i=2^{k}-2^{j-1} p=2^{j-1}\left(2^{k-j+1}-p\right)$ and $2^{k-j+1}-p$ is odd. Note that neither $i$ nor $2^{k}-i$ admits $2^{k}$ as a divisor; thus

$$
b_{i}=a_{i}=\frac{1}{2^{j-1}}=a_{2^{j-1}\left(2^{k-j+1}-p\right)}=a_{2^{k}-i}=b_{2^{k}-i} .
$$

By the above claim, one can see that $T$ is unitarily equivalent to $A^{(\infty)}$, the orthogonal direct sum of $\aleph_{0}$ copies of $A$, where $A \in \mathcal{B}\left(\mathbb{C}^{2^{k}}\right)$ and

$$
A=\left[\begin{array}{ccccc}
0 & & & & \\
b_{1} & 0 & & & \\
& b_{2} & 0 & & \\
& & \ddots & \ddots & \\
& & & b_{2^{k}-1} & 0
\end{array}\right] \begin{gathered}
f_{1} \\
f_{2} \\
f_{3} \\
\vdots \\
f_{2^{k}}
\end{gathered}
$$

Here $\left\{f_{i}\right\}_{i=1}^{2^{k}}$ is an ONB of $\mathbb{C}^{2^{k}}$.

Note that $b_{i}=b_{2^{k}-i}$ for all $1 \leq i \leq 2^{k}-1$. Then, by Proposition 0.4 , $A^{*}$ and $A$ are both complex symmetric. Since $\mathcal{S}(\mathcal{H})$ is invariant under unitary equivalence, it follows that $T$ is also complex symmetric. Thus we conclude the proof.

Remark 0.6. Note that $\operatorname{dim} \operatorname{ker} W^{*}=1$ and $\operatorname{ker} W=\{0\}$. Then one can immediately deduce that $W$ is not complex symmetric (see [4, Proposition 1]). It follows from Theorem 0.5 that the class $\mathcal{S}(\mathcal{H})$ of complex symmetric operators is not norm closed.

Since $\mathcal{S}(\mathcal{H})$ is not norm closed, it is natural and interesting to consider the following question.

Question 0.7. What is the norm closure of $\mathcal{S}(\mathcal{H})$ ?

\section{REFERENCES}

1. L. Balayan and S. R. Garcia, Unitary equivalence to a complex symmetric matrix: Geometric criteria, Oper. Matrices 4 (2010), no. 1, 53-76. MR2655004

2. J. A. Cima, S. R. Garcia, W. T. Ross, and Warren R. Wogen, Truncated Toeplitz operators: Spatial isomorphism, unitary equivalence, and similarity, Indiana Univ. Math. J. 59 (2010), no. 2, 595-620. MR 2648079

3. S. R. Garcia, Aluthge transforms of complex symmetric operators, Integral Equations Operator Theory 60 (2008), no. 3, 357-367. MR2392831 (2008m:47052) 
4. S. R. Garcia and M. Putinar, Complex symmetric operators and applications, Trans. Amer. Math. Soc. 358 (2006), no. 3, 1285-1315 (electronic). MR2187654 (2006j:47036)

5. __ Complex symmetric operators and applications. II, Trans. Amer. Math. Soc. 359 (2007), no. 8, 3913-3931 (electronic). MR2302518(2008b:47005)

6. I__ Interpolation and complex symmetry, Tohoku Math. J. (2) 60 (2008), no. 3, 423-440. MR2453732 (2009k:47048)

7. S. R. Garcia and W. R. Wogen, Complex symmetric partial isometries, J. Funct. Anal. 257 (2009), no. 4, 1251-1260. MR2535469

8. _ Some new classes of complex symmetric operators, Trans. Amer. Math. Soc. 362 (2010), no. 11, 6065-6077. MR2661508

9. T. M. Gilbreath and W. R. Wogen, Remarks on the structure of complex symmetric operators, Integral Equations Operator Theory 59 (2007), no. 4, 585-590. MR2370050 (2009f:47037)

10. C. E. Rickart, General theory of Banach algebras, The University Series in Higher Mathematics, D. van Nostrand Co., Inc., Princeton, N.J.-Toronto-London-New York, 1960. MR0115101 $(22: 5903)$

Department of Mathematics, Jilin University, Changchun 130012, People's Republic OF CHINA

Current address: School of Mathematical Sciences, Fudan University, 220 Handan Road, Shanghai 200433, People's Republic of China

E-mail address: zhusen@jlu.edu.cn

Institute of Mathematics, Jilin University, Changchun 130012, People's Republic of CHINA

E-mail address: licg09@mails.jlu.edu.cn

Department of Mathematics, Jilin University, Changchun 130012, People's Republic OF CHINA

E-mail address: jiyq@jlu.edu.cn 